\title{
Preclinical Neonatal Rat Studies of Heparin-Binding EGF-Like Growth Factor in Protection of the Intestines From Necrotizing Enterocolitis
}

\author{
ANDREI RADULESCU, NICHOLAS A. ZORKO, XIAOYI YU, AND GAIL E. BESNER
}

Center for Perinatal Research [A.R., X.Y., G.E.B.], Department of Pediatric Surgery [G.E.B.], Nationwide Children's Hospital, Columbus, Ohio, 43205; College of Medicine [N.A.Z., G.E.B.], The Ohio State University, Columbus, Ohio 43210

\begin{abstract}
We have previously demonstrated that enterally administered heparin-binding EGF-like growth factor (HB-EGF) produced in Escherichia coli decreases the incidence and severity of intestinal injury in a neonatal rat model of necrotizing enterocolitis (NEC). In preparation for upcoming human clinical trials, large-scale production of HB-EGF according to Good Manufacturing Practice (GMP) has been successfully accomplished using a Pichia pastoris yeast system. The current studies used a neonatal rat model of NEC to elucidate several important preclinical characteristics of HB-EGF therapy. We found that enteral administration of HB-EGF (800 $\mu \mathrm{g} / \mathrm{kg} / \mathrm{dose}$ ) four times a day effectively reduced the incidence and severity of NEC, that Pichia-derived HB-EGF was not significantly different from E. coli-derived HB-EGF in preventing NEC, that EGF was not superior to HB-EGF in preventing NEC, and that prophylactic administration of HB-EGF added to formula starting with the first feed or $12 \mathrm{~h}$ later significantly reduced the incidence of NEC, with no change in the incidence of NEC noted if HB-EGF was added to the formula starting 24,48 , or $72 \mathrm{~h}$ after birth. Thus, large-scale production of GMP-grade HB-EGF in Pichia pastoris yeast produces a biologically active molecule suitable for human clinical trials. (Pediatr Res 65: 437-442, 2009)
\end{abstract}

$\mathrm{N}$ ecrotizing enterocolitis (NEC) is the most common gastrointestinal emergency in premature newborn infants $(1,2)$. With aggressive management leading to the salvage of premature infants from the pulmonary standpoint, the incidence of NEC is increasing, and is expected to soon replace pulmonary insufficiency as the leading cause of death in premature infants (3). NEC mortality ranges from 20 to $50 \%$, resulting in over 1000 deaths in this country each year. Survivors frequently develop malabsorption, malnutrition, total parenteral nutrition-related complications, intestinal strictures, and short bowel syndrome (4).

Because prematurity is the single most important risk factor for NEC, it is possible that absent or reduced levels of specific factors that are normally expressed during later periods of gestation may contribute to the development of this condition.

Received September 29, 2008; accepted November 25, 2008.

Correspondence: Gail E. Besner, M.D., Nationwide Children's Hospital, Department of Surgery ED 321, 700 Children's Drive, Columbus, OH 43205; e-mail: Gail.Besner@NationwideChildrens.org

Supported by Trillium Therapeutics, Inc. (Toronto, Ontario, Canada) (G.E.B., A.R.), NIH RO1 DK074611 (G.E.B.), NIHT32 RR-023260-02 (N.A.Z.), The Samuel J. Roessler Memorial Fellowship (N.A.Z.), and The Firefighters Endowment Fund of Nationwide Children's Hospital (X.Y.).
We have previously examined HB-EGF mRNA expression in intestine resected from patients with NEC, comparing intestinal samples from areas afflicted with acute NEC with the more normal intestine at the resection margins. We found that NEC-afflicted intestine had significantly lower HB-EGF mRNA levels compared with intestine at the resection margins (5). We speculated that a relative deficiency of HB-EGF in the NEC-afflicted intestine might reflect part of the pathogenesis of NEC. With this in mind, exogenous replacement of HBEGF may be clinically valuable as a means to reduce the incidence of NEC. Although many potential preventive strategies for NEC have been examined in the past, none have led to consistently positive therapeutic results (5). Presently, no clinical trials have addressed the use of growth factors as intestinal cytoprotective agents for NEC.

Heparin-binding EGF-like growth factor (HB-EGF) was initially identified in the conditioned medium of cultured human macrophages (6) and later found to be a member of the EGF family of growth factors (7). HB-EGF exerts its mitogenic effects by binding and activation of EGF receptor subtypes ErbB-1 and ErbB-4 (8). In addition, HB-EGF exerts chemotactic effects when binding to the HB-EGF-specific receptor $\mathrm{N}$-arginine dibasic convertase ( $\mathrm{NrdC})(9)$. Importantly, endogenous HB-EGF is protective in various pathologic conditions and plays a pivotal role in mediating the earliest cellular responses to proliferative stimuli and cellular injury.

We have previously demonstrated that enteral administration of Escherichia coli-derived HB-EGF decreases the incidence and severity of intestinal injury in a neonatal rat model of NEC, with the greatest protective effects found at doses of 600 or $800 \mu \mathrm{g} / \mathrm{kg} / \mathrm{dose}(10)$. We have also reported that HB-EGF protects the intestines from injury after intestinal ischemia/reperfusion (11) or hemorrhagic shock and resuscitation (12). Given the evidence to date demonstrating its intestinal protective effects, HB-EGF may represent a promising therapeutic strategy for intestinal diseases including NEC. In preparation for upcoming human clinical trials of HB-EGF for the prevention of NEC, we have produced human recombinant mature HB-EGF according to Good Manufactur-

\footnotetext{
Abbreviations: EGFR, epidermal growth factor receptor; GMP, good manufacturing process; HB-EGF, heparin-binding EGF-like growth factor; NEC, necrotizing enterocolitis
} 
ing Process (GMP) using a high-yield Pichia pastoris yeast expression system. The goals of the current project were to determine the optimal HB-EGF dosing interval, to examine the efficacy of Pichia-derived GMP-grade HB-EGF compared with $E$. coli-derived HB-EGF, to evaluate the efficacy of HB-EGF and EGF in preventing NEC, and to compare prophylactic and therapeutic administration of HB-EGF in the prevention of NEC.

\section{MATERIALS AND METHODS}

Neonatal rat model of experimental necrotizing enterocolitis. The experimental protocol was performed according to the guidelines for the ethical treatment of experimental animals and approved by our Institutional Animal Care and Use Committee (\#04203AR). NEC was induced using a modification of the model described by Barlow et al. (13). Pregnant time-dated Sprague-Dawley rats (Harlan Sprague-Dawley, Indianapolis, IN) were delivered by $\mathrm{C}$-section under $\mathrm{CO}_{2}$ anesthesia on day 21.5 of gestation. Newborn rats were placed in an incubator and fed via gavage with formula containing 15 g Similac 60/40 (Ross Pediatrics, Columbus, OH) in $75 \mathrm{~mL}$ Esbilac (Pet-Ag, New Hampshire, IL), providing $836.8 \mathrm{~kJ} / \mathrm{kg}$ per day. Feeds were started at $0.1 \mathrm{~mL}$ every $4 \mathrm{~h}$ beginning $2 \mathrm{~h}$ after birth and advanced as tolerated up to a maximum of $0.4 \mathrm{~mL}$ per feeding by the fourth day of life. Animals were exposed to a single dose of intragastric lipopolysaccharide (LPS; 2 $\mathrm{mg} / \mathrm{kg}) 8 \mathrm{~h}$ after birth, and were stressed by exposure to hypoxia (100\% nitrogen for $1 \mathrm{~min}$ ) followed by hypothermia $\left(4^{\circ} \mathrm{C}\right.$ for $\left.10 \mathrm{~min}\right)$ twice a day beginning immediately after birth until the end of the experiment.

To investigate HB-EGF dosing interval, 203 rat pups were randomized to receive HB-EGF added to their feeds two (BID), three (TID), four (QID), or six (HID) equally spaced times a day. To compare the efficacy of $E$. coli-derived and P. pastoris-derived HB-EGF, 199 pups were randomized to receive 600,800 , or $1000 \mu \mathrm{g} / \mathrm{kg} / \mathrm{dose}$ of each type of HB-EGF added to their feeds QID or HID. To compare the efficacy of HB-EGF and EGF in the prevention of NEC, 120 pups were randomized to receive either equal mass doses of each growth factor (HB-EGF $800 \mu \mathrm{g} / \mathrm{kg} / \mathrm{dose}$ versus EGF 800 $\mu \mathrm{g} / \mathrm{kg} / \mathrm{dose}$ ) or molar equivalents of each growth factor (HB-EGF 800 $\mu \mathrm{g} / \mathrm{kg} / \mathrm{dose}$ versus EGF $570 \mu \mathrm{g} / \mathrm{kg} /$ dose). To compare prophylactic and therapeutic administration of HB-EGF in NEC, 137 pups were exposed to stress beginning immediately after birth, with HB-EGF $(800 \mu \mathrm{g} / \mathrm{kg} / \mathrm{dose})$ added to the feeds starting at $2,12,24,48$, or $72 \mathrm{~h}$ after birth.

The HB-EGF used in all experiments was GMP grade human mature HB-EGF produced in $P$. pastoris yeast and supplied to us by Trillium Therapeutics, Toronto, Canada. E. coli-derived recombinant human mature HB-EGF was produced in our laboratory as previously described (14). EGF was produced in E. coli and purchased from Vybion (Ithaca, NY). In all experiments, pups were euthanized on development of clinical signs of NEC (abdominal distention, bloody bowel movements, respiratory distress, lethargy). Remaining animals were killed $96 \mathrm{~h}$ after birth.

Histologic Injury Score. On sacrifice, the gastrointestinal tract was carefully removed and visually evaluated for signs of NEC (areas of bowel necrosis, intestinal hemorrhage, perforation). Three pieces of duodenum, jejunum, ileum, and colon from every animal were fixed in $10 \%$ formalin for $24 \mathrm{~h}$, paraffin-embedded, sectioned at $5 \mu \mathrm{m}$ thickness, and stained with hematoxylin and eosin for histologic evaluation of the presence and/or degree of NEC using the NEC histologic injury scoring system described by Caplan et al. (15). Histologic changes were graded as follows: grade 0, no damage; grade 1, epithelial cell lifting or separation; grade 2, sloughing of epithelial cells to the mid villus level; grade 3, necrosis of the entire villus; and grade 4 , transmural necrosis. Tissues were graded blindly by two independent observers. Tissues with histologic scores of 2 or higher were considered positive for NEC (Fig. 1).

Statistical analyses. Fisher's exact test was used for comparing the incidence of NEC between groups with no adjustments made for multiple comparisons. $p$-values less then 0.05 were considered statistically significant. All statistical analyses were performed using SAS (version 9.1,SAS Institute, Cary, NC).

\section{RESULTS}

Dosing interval of HB-EGF administration. We have previously shown that enteral administration of HB-EGF at doses of 600 or $800 \mu \mathrm{g} / \mathrm{kg} /$ dose administered HID significantly

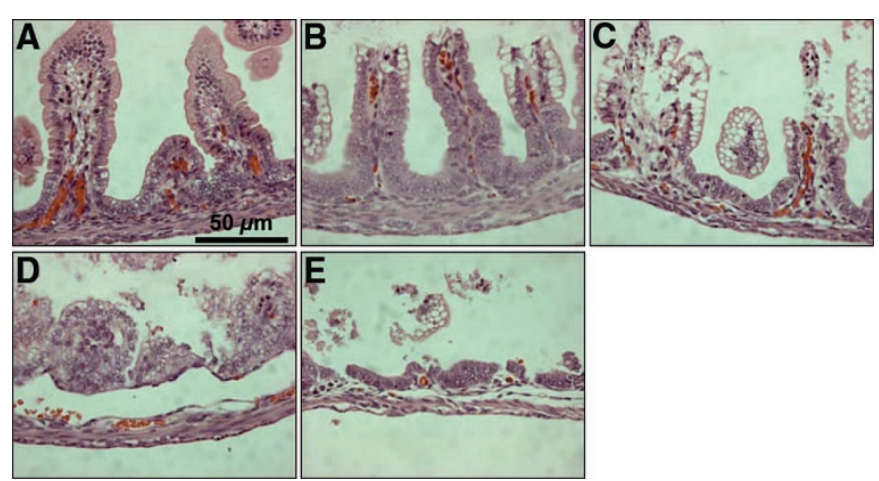

Figure 1. Histologic injury score in rat pups subjected to experimental NEC. Shown are representative H\&E-stained sections showing $(A)$ normal intestine, $(B)$ Grade 1, epithelial cell lifting or separation, $(C)$ Grade 2, sloughing of epithelial cells to the mid villous level, $(D)$ Grade 3, necrosis of the entire villus, and $(E)$ Grade 4, transmural necrosis. Magnification $\times 40$.

decreases the incidence and severity of experimental NEC (10). However, we did not examine whether administration less frequently than HID could also protect the intestines from NEC. In this experiment, we examined the effect of decreasing HB-EGF dosing intervals. Animals subjected to stress had a $63 \%$ incidence of NEC, with histopathologic changes ranging from moderate, mid-level villous necrosis (grade 2 ) to severe necrosis of the entire villous (grade 3 and 4) (Fig. $2 A$ and $B$ ). Rat pups that received HB-EGF ( $800 \mu \mathrm{g} / \mathrm{kg} / \mathrm{dose})$ added to every feed (HID) showed a significant decrease in the incidence of NEC to $39 \%$ ( $p=0.03$ ). We found that decreasing the HB-EGF dosing interval to either BID or QID also significantly reduced the percent of animals that developed NEC to $38 \%$ and $22 \%$, respectively ( $p=0.05$ and $p<0.001$ ).

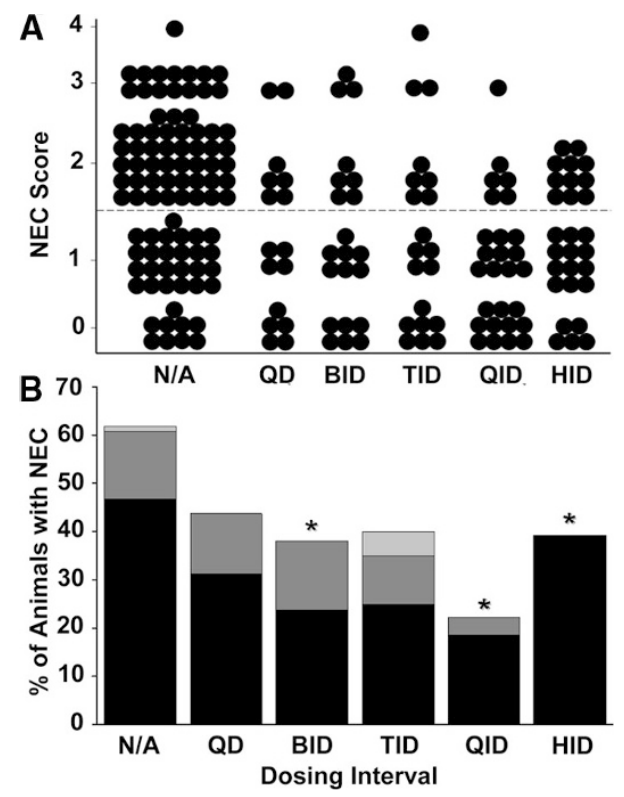

Figure 2. HB-EGF dosing interval. (A) NEC Score. The effect of HB-EGF (800 $\mu \mathrm{g} / \mathrm{kg} / \mathrm{dose}$ ) added to feeds one (QD), two (BID), three (TID), four (QID), or six (HID) times a day on the development of NEC is shown. Each dot represents a single rat pup exposed to NEC, and the NEC score for each pup is shown. $(B)$ Incidence of NEC. The percent of animals with NEC at each dosing interval is shown. ${ }^{*} p<0.05$ compared with the non-HB-EGF-treated control group. N/A, no addition of HB-EGF to feeds. Grade 2 NEC, $\mathbf{\square}$; grade 3 NEC, $\square$; grade 4 NEC, $\square$. 
There was no statistically significant difference between administration of HB-EGF HID or QID. Although there was a trend toward an increased number of animals with grade 2 NEC in the HID group, there were no statistically significant differences between QID and HID dosing when comparing animals with grade 2 NEC ( $p=0.24)$.

In addition to decreasing the incidence of NEC, we also noted that addition of HB-EGF to the feeds decreased the degree of intestinal damage in the pups that did develop NEC. In non-HB-EGF-treated pups, of the $63 \%$ of pups that developed NEC, $1.7 \%$ had grade 4 injury, $24.1 \%$ had grade 3 injury, and $74.1 \%$ had grade 2 injury. However, in pups treated with HBEGF QID, of the $22 \%$ that did develop NEC, only $16.6 \%$ had grade 3 injury, and $83.3 \%$ had grade 2 injury. Furthermore, we found that $\sim 15 \%$ of non-HB-EGF-treated animals survived the 96-h protocol without developing clinical signs of NEC, but with the addition of HB-EGF to the feeds QID or HID, survival significantly increased to $\sim 80 \%$ (data not shown).

Comparison of $\mathrm{P}$. pastoris-derived and $\mathrm{E}$. coli-derived $\boldsymbol{H B}-\boldsymbol{E} \boldsymbol{G} \boldsymbol{F}$. We have produced recombinant GMP-grade HBEGF in quantities sufficient for human clinical trials using a $P$. pastoris yeast expression system. We next tested the efficacy of $P$. pastoris-derived HB-EGF compared with E. coli-derived HB-EGF in preventing NEC. Because our previous studies of E. coli-derived HB-EGF in preventing NEC tested doses up to but not exceeding $800 \mu \mathrm{g} / \mathrm{kg} / \mathrm{dose}$, we also tested whether increasing the dose of HB-EGF to $1000 \mu \mathrm{g} / \mathrm{kg} /$ dose would lead to further beneficial effects. In this experiment, the incidence of NEC in stressed pups was $68 \%$. When tested at doses of 600,800 , or $1000 \mu \mathrm{g} / \mathrm{kg} /$ dose, and dosing intervals of QID or HID, there were no significant differences in efficacy between E. coli-derived and Pichia-derived HB-EGF (Fig. 3A and $B$ ). Although there was a trend toward a decreased incidence of NEC in animals that received Pichia-derived HB-EGF HID compared with QID, there were no statistically significant differences between the two groups at either of the three doses of HB-EGF tested.

Comparison of HB-EGF and EGF. To accurately compare the efficacy of HB-EGF and EGF in preventing NEC, we chose a dose of HB-EGF (800 $\mu \mathrm{g} / \mathrm{kg} / \mathrm{dose})$ with proven efficacy in preventing NEC in our hands, and compared this dose to both the equivalent mass dose $(800 \mu \mathrm{g} / \mathrm{kg} / \mathrm{dose})$ and the equivalent molar dose of EGF (570 $\mu \mathrm{g} / \mathrm{kg} / \mathrm{dose})$. Comparing equal molar doses takes into account the different molecular masses of the mature forms of the two growth factors (i.e. HB-EGF residues 74-148; [74aa; Mr7400] versus EGF residues 1-53 [53aa; Mr 5300]), and adds an equal number of molecules of each growth factor to the experiment. In this experiment, animals subjected to stress had an incidence of NEC of $63.3 \%$ (Fig. $4 A$ and $B$ ). HB-EGF (800 $\mu \mathrm{g} / \mathrm{kg} / \mathrm{dose}$ ) significantly decreased the incidence of NEC to $30.7 \%$ ( $p=$ 0.009). The equivalent mass dose of EGF ( $800 \mu \mathrm{g} / \mathrm{kg} / \mathrm{dose})$ significantly decreased the incidence of NEC to $21.7 \%$ ( $p=$ $0.002)$, and the equivalent molar dose $(570 \mu \mathrm{g} / \mathrm{kg} / \mathrm{dose}) \mathrm{de}-$ creased the incidence to $40.9 \%(p=0.12)$. There were no statistically significant differences in the incidence of NEC between HB-EGF and either of the two doses of EGF tested.

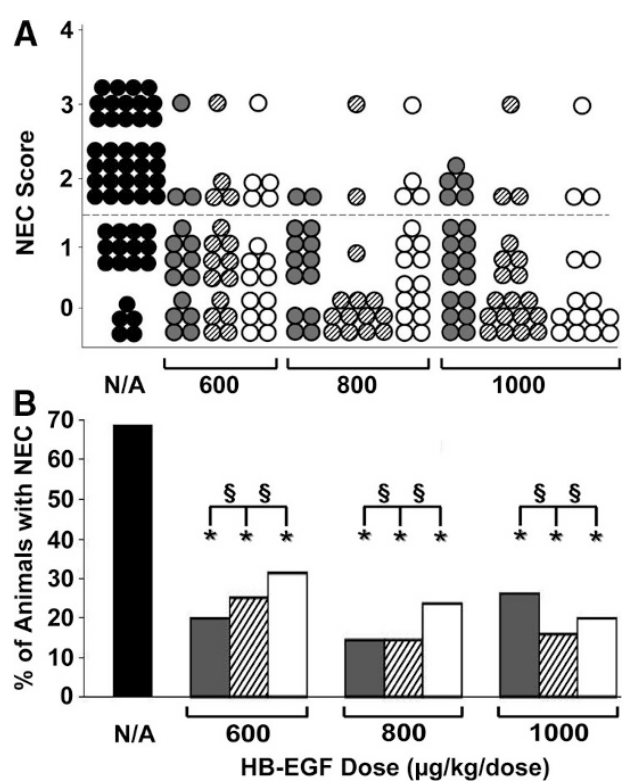

Figure 3. Comparison of E. coli- and P. pastoris-derived HB-EGF in prevention of NEC. (A) NEC Score. Recombinant HB-EGF derived from $E$. coli or $P$. pastoris was tested at doses of 600,800 , or $1000 \mu \mathrm{g} / \mathrm{kg} / \mathrm{dose}$, added to feeds either QID or HID. Each dot represents a single rat pup exposed to NEC, and the NEC score for each pup is shown. No addition of HB-EGF, $\mathbf{O}$; coli-derived HB-EGF HID, O; $P$. pastoris-derived HB-EGF HID, $\oslash ; P$. pastoris-derived HB-EGF QID, $\bigcirc$. (B) Incidence of NEC. The percent of animals with NEC in pups that were treated with E. coli- or P. pastorisderived HB-EGF is shown. No addition of HB-EGF, $\mathbf{\square}$; E. coli-derived HB-EGF HID, $\square$; $P$. pastoris-derived HB-EGF HID, 凤; P. pastoris-derived HB-EGF QID, $\square . * p<0.05$ compared with the non-HB-EGF-treated control group; §, not significant; N/A, no addition of HB-EGF.

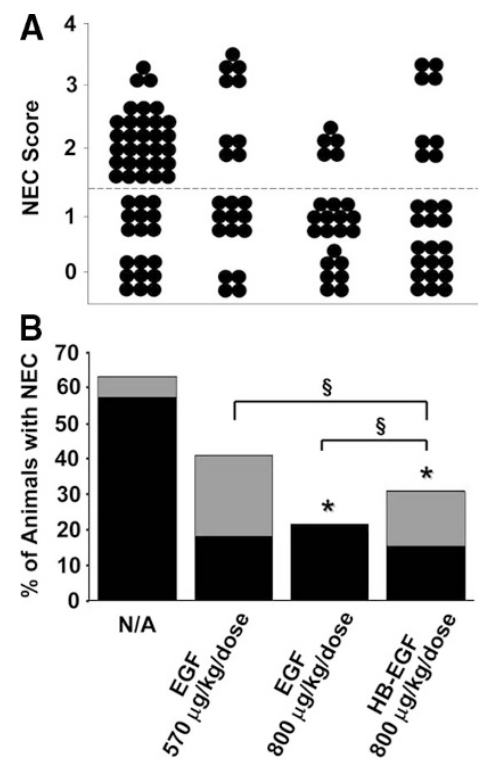

Figure 4. Comparison of HB-EGF and EGF in prevention of NEC. (A) NEC Scores. Either equal molar $(800 \mu \mathrm{g} / \mathrm{kg} / \mathrm{dose} \mathrm{HB}-\mathrm{EGF}$ versus $570 \mu \mathrm{g} / \mathrm{kg} / \mathrm{dose}$ EGF) or equal mass ( $800 \mu \mathrm{g} / \mathrm{kg} /$ dose HB-EGF versus $800 \mu \mathrm{g} / \mathrm{kg} / \mathrm{dose}$ EGF) amounts of HB-EGF and EGF were compared in their ability to prevent NEC. Each dot represents a single rat pup exposed to experimental NEC, and the NEC score for each pup is shown. (B) Incidence of NEC. The percent of animals with NEC in pups that received either equal molar or equal mass amounts of HB-EGF or EGF is shown. ${ }^{*} p<0.05$ compared with the non-growth factor-treated control group; §, not significant; N/A, no addition of HB-EGF to feeds. Grade 2 NEC, $\mathbf{\square}$; grade 3 NEC, 
Comparison of prophylactic and therapeutic administration of HB-EGF. In the future, clinical administration of HB-EGF for NEC could be performed prophylactically in an attempt to prevent NEC from developing, or therapeutically in an attempt to reverse or inhibit the progression of NEC. We previously used a rodent model of intestinal ischemia/ reperfusion injury secondary to superior mesenteric artery occlusion to show that HB-EGF can significantly protect the intestines from injury when administered either prophylactically or therapeutically; however, the best results were obtained when HB-EGF was administered before injury (16). Similar experiments using the rodent model of NEC have not been previously performed. We therefore subjected rat pups to stress beginning immediately after birth, with addition of HB-EGF (800 $\mu \mathrm{g} / \mathrm{kg} / \mathrm{dose})$ to the feeds beginning with the first feed at $2 \mathrm{~h}$ after birth, or beginning after 12, 24, 48, or $72 \mathrm{~h}$ after birth. In this experiment, the incidence of NEC in stressed animals was $67.3 \%$ (Fig. 5). The incidence of NEC decreased significantly to $26.3 \%$ when HB-EGF was added to the feeds starting at $2 \mathrm{~h}$, and to $25.0 \%$ when HB-EGF was started $12 \mathrm{~h}$ after birth $(p=0.003$ and $p=0.001$, respectively). In addition to decreasing the incidence of NEC, we also noted that HB-EGF supplementation at the $2 \mathrm{~h}$ or $12 \mathrm{~h}$ time points decreased the degree of intestinal damage in the pups that did develop NEC. Of the $67.3 \%$ of stressed animals that developed NEC, $78.8 \%$ had grade 2 injury, and $21.2 \%$ had grade 3 injury. In animals that received HB-EGF starting $2 \mathrm{~h}$ after birth, of the $26.3 \%$ that went on to develop NEC, only $20 \%$ had grade 3 injury and $80 \%$ had grade 2 injury. In pups that received HB-EGF starting $12 \mathrm{~h}$ after birth, of the $25 \%$ that

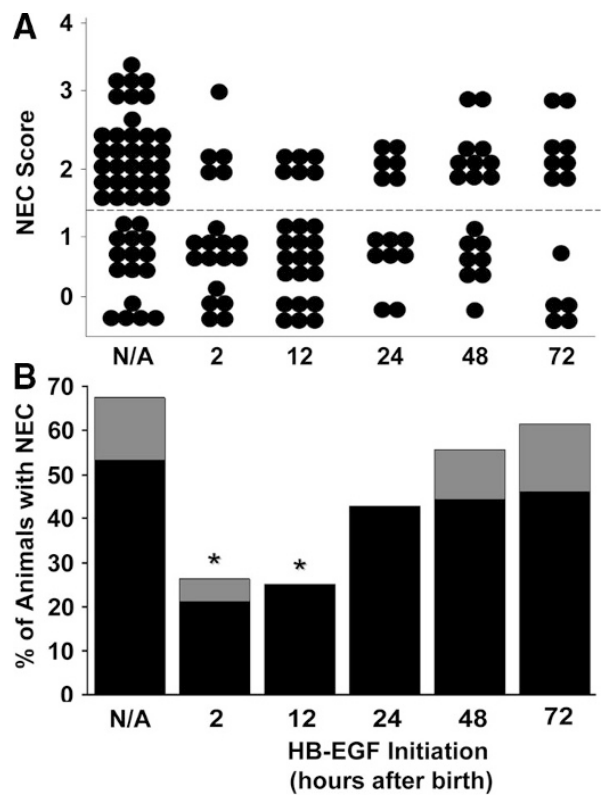

Figure 5. Comparison of prophylactic and therapeutic administration of HB-EGF. (A) NEC Scores. The effect of HB-EGF ( $800 \mu \mathrm{g} / \mathrm{kg} / \mathrm{dose})$ added to feeds starting with the first feed at $2 \mathrm{~h}$ after birth, or at $12,24,48$, or $72 \mathrm{~h}$ after birth is shown. Each dot represents a single rat pup exposed to experimental NEC, and the NEC score for each pup is shown. (B) Incidence of NEC. The percent of animals with NEC in pups that received HB-EGF ( $800 \mu \mathrm{g} / \mathrm{kg} / \mathrm{dose})$ starting $2,12,24,48$, or $72 \mathrm{~h}$ after birth is shown. ${ }^{*} p<0.05$ compared with the non-HB-EGF-treated control group; N/A, no addition of HB-EGF. Grade 2 NEC, $\square$; grade 3 NEC, $\square$. went on to develop NEC, none had grade 3 injury and $100 \%$ had grade 2 injury. When HB-EGF administration was started at later time points $(24,48$, and $72 \mathrm{~h})$, there were no significant differences in the incidence or severity of NEC compared with control animals.

We recently examined the time course for development of NEC in our NEC model, and have determined that clinical signs and intestinal histologic damage consistent with NEC are noted beginning at $48-72 \mathrm{~h}$ of stress (data not shown). Here we show that administration of HB-EGF starting at 2 or $12 \mathrm{~h}$ of stress significantly decreased the incidence of NEC, administration starting at $24 \mathrm{~h}$ of stress decreased the incidence of NEC to a lesser degree, and administration starting at 48 or $72 \mathrm{~h}$ of stress does not significantly decrease the incidence of NEC. This suggests that once intestinal necrosis has already occurred in these animals, HB-EGF is less effective in preventing NEC. Thus, these data suggest that prophylactic rather than therapeutic use of HB-EGF is more effective in this animal model.

\section{DISCUSSION}

Although many clinical trials have been conducted in an attempt to protect newborns from NEC, clinical trials of growth factors in the prevention of NEC have never been performed. We have used several different intestinal injury models to show that HB-EGF protects the intestines from injury $(5,10-12)$. We have also shown that HB-EGF is present in human amniotic fluid and breast milk, ensuring continuous natural exposure of the fetal and newborn intestine to endogenous levels of the growth factor before and after birth (17). Supplementation of enteral feeds with a biologically active substance such as HB-EGF may represent a logical and safe way to reduce intestinal injury resulting in NEC. HB-EGF administration to very low birth weight patients $(<1500 \mathrm{~g})$ who are most at risk for developing NEC may facilitate maturation, enhance regenerative capacity, and increase resistance of the intestinal mucosa to injury.

We have previously shown that intragastric administration of ${ }^{125}$ I-labeled HB-EGF to rats leads to delivery of the growth factor to the entire GI tract including the colon within $8 \mathrm{~h}$ (18). We found peak HB-EGF concentrations in the plasma $4 \mathrm{~h}$ after dosing, with a half-life in the absorption phase of $2.38 \mathrm{~h}$ and in the elimination phase of $11.13 \mathrm{~h}$. As part of our extensive recent preclinical studies of HB-EGF, single-dose and repeat-dose toxicology studies have been performed in neonatal and adult Sprague Dawley rats and Yukatan minipigs. HB-EGF was administered enterally for up to $28 \mathrm{~d}$, at doses up to 70 times higher than the doses effective in our rat NEC model. Even at the highest doses, there were no toxic or adverse effects detected, suggesting that there is an excellent safety margin for HB-EGF administration (unpublished data). In addition, there was no systemic absorption detected with enteral administration of HB-EGF, even at the highest doses administered. These findings collectively support the clinical feasibility and safety of enteral administration of HB-EGF in protection of the intestines from injury. The results reported here, involving HB-EGF dosage, dosing interval, and efficacy 
of GMP-grade HB-EGF, adds important preclinical information to the design of future clinical trials of HB-EGF in NEC.

EGF family members are of interest as intestinal protective agents because of their roles in gut maturation and function. Infants with NEC have decreased levels of salivary EGF, as do very premature infants $(19,20)$. EGF preserves gut barrier function, increases intestinal enzyme activity, and improves nutrient transport (21). EGF receptor (EGFR) knockout mice develop epithelial cell abnormalities and hemorrhagic necrosis of the intestine similar to neonatal NEC, suggesting that lack of EGFR stimulation may play a role in the development of NEC (22). Dvorak et al. have shown that EGF supplementation reduces the incidence of NEC in rats, and reduces apoptosis, barrier failure, and hepatic dysfunction (23). VinterJensen et al., investigated the effect of s.c. administered EGF $(150 \mu \mathrm{g} / \mathrm{kg} / 12 \mathrm{~h})$ in rats, for 1,2 , and $4 \mathrm{wk}$, and found that EGF induced growth of small intestinal mucosa and muscularis in a time-dependent manner (24). Several case reports of clinical administration of EGF also exist. Sigalet et al. administered EGF (100 $\mu \mathrm{g} / \mathrm{kg} / \mathrm{d})$ mixed with enteral feeds for $6 \mathrm{wk}$ to pediatric patients with short bowel syndrome, and reported improved nutrient absorption and increased tolerance to enteral feeds with no adverse effects (25). Sullivan et al., in a prospective, double-blind, randomized controlled study that included eight neonates with NEC, compared the effects of a 6- $\mathrm{d}$ continuous i.v. infusion of EGF (100 ng/kg/h) to placebo, and found a positive trophic effect of EGF on the intestinal mucosa (26). Palomino et al. examined the efficacy of EGF in the treatment of duodenal ulcers in adults, and found that oral human recombinant EGF ( $50 \mathrm{mg} / \mathrm{mL}$ every $8 \mathrm{~h}$ for $6 \mathrm{wk}$ ) was effective with no side effects noted (27).

In a recent report, Dvorak et al. compared the effect of enteral administration of HB-EGF compared with EGF in protection from NEC in newborn rats (28). The authors concluded that both growth factors could protect rat pups from developing NEC, but suggested that EGF may be effective at more physiologic levels. The basis of that conclusion is not totally clear, because both growth factors in their study had maximal beneficial effects at the same dose $(500 \mathrm{ng} / \mathrm{mL})$. There are several difficulties encountered when comparing the results of the Dvorak study with ours. First, Dvorak et al. report their growth factor doses in $\mathrm{ng} / \mathrm{mL}$ rather than in $\mathrm{ng}$ or $\mu \mathrm{g} / \mathrm{kg} / \mathrm{dose}$. We dose our rat pups in $\mu \mathrm{g} / \mathrm{kg} / \mathrm{dose}$ because this is directly comparable to the doses pediatric patients receive clinically, and because this allows us to determine the human equivalent dose of HB-EGF using the following formula (29):

$\mathrm{HED}=$ animal dose in $\mathrm{mg} / \mathrm{kg} \times$ [animal weight in $\mathrm{kg} /$

$$
\text { human weight in } \mathrm{kg}]^{0.33}
$$

Furthermore, Dvorak et al. never state the volume (mL) of the feeds that were administered, or the number of doses that were administered daily, making it impossible to definitively determine the exact amounts of growth factors administered. However, if we assume that they administered $0.1-0.4 \mathrm{~mL} /$ feed, and that their newborn rat pups weighed $\sim 0.005 \mathrm{~kg}$, then they delivered $\sim 10-40 \mu \mathrm{g} / \mathrm{kg} /$ dose of HB-EGF or EGF in their experiments, which is $\sim 20$-fold less HB-EGF than the most efficacious dose of HB-EGF in our hands. In fact, using the NEC injury grading system that we use [as proposed by Caplan et al. (15)] we would not see any beneficial effects of HB-EGF at the doses used by Dvorak et al. This may be because different injury scoring systems are used in the two studies. Although they do not show any histologic images in their recent paper, previous studies from their laboratory have shown histology (23). What they judge as grade 2 or 3 NEC would not meet the criteria for NEC using our scoring system. What they judge as grade $4 \mathrm{NEC}$ is comparable to what we would judge as grade 2 NEC (the minimal degree of injury required to be considered NEC). We believe that the more severe degrees of injury we are producing in our animal model are more comparable to the clinically relevant, severe structural destruction of the intestines seen in patients afflicted with NEC. Future clinical trials of HB-EGF in humans will be needed to definitively elucidate the doses of HB-EGF that are truly needed to prevent or treat this devastating disease.

Our goal is the clinical use of HB-EGF in the prevention or treatment of NEC. Given the evidence demonstrating the protective effects of HB-EGF against intestinal injury, its use may represent a promising therapeutic strategy for intestinal diseases, including NEC. Because of its ability to enhance the regenerative capacity and/or increase the resistance of the mucosa to injury, HB-EGF has proven to be a strong candidate for human clinical trials for NEC.

Acknowledgments. The authors wish to thank Gregory Young, M.S., Ohio State University Center for Biostatistics, for his helpful comments and his assistance with the statistical analyses.

\section{REFERENCES}

1. Schnabl KL, Van Aerde JE, Thomson AB, Clandinin MT 2008 Necrotizing enterocolitis: a multifactorial disease with no cure. World J Gastroenterol 14:2142-2161

2. Kliegman RM, Fanaroff AA 1984 Necrotizing enterocolitis. N Engl J Med 310:1093-1103

3. Lee JS, Polin RA 2003 Treatment and prevention of necrotizing enterocolitis. Semin Neonatol 8:449-459

4. Caplan MS, Jilling T 2001 New concepts in necrotizing enterocolitis. Curr Opin Pediatr 13:111-115

5. Feng J, El-Assal ON, Besner GE 2005 Heparin-binding EGF-like growth factor (HB-EGF) and necrotizing enterocolitis. Semin Pediatr Surg 14:167-174

6. Besner G, Higashiyama S, Klagsbrun M 1990 Isolation and characterization of a macrophage-derived heparin-binding growth factor. Cell Regul 1:811-819

7. Higashiyama S, Abraham JA, Miller J, Fiddes JC, Klagsbrun M 1991 A heparinbinding growth factor secreted by macrophage-like cells that is related to EGF. Science 251:936-939

8. Junttila TT, Sundvall M, Määttä JA, Elenius K 2000 Erbb4 and its isoforms: selective regulation of growth factor responses by naturally occurring receptor variants. Trends Cardiovasc Med 10:304-310

9. Nishi E, Prat A, Hospital V, Elenius K, Klagsbrun M $2001 \mathrm{~N}$-arginine dibasic convertase is a specific receptor for heparin-binding EGF-like growth factor that mediates cell migration. EMBO J 20:3342-3350

10. Feng J, El-Assal ON, Besner GE 2006 Heparin-binding epidermal growth factor-like growth factor decreases the incidence of necrotizing enterocolitis in neonatal rats. J Pediatr Surg 41:144-149

11. El-Assal ON, Besner GE 2004 Heparin-binding EGF-like growth factor (HB-EGF) and intestinal ischemia-reperfusion injury. Semin Pediatr Surg 13:2-10

12. El-Assal ON, Radulescu A, Besner GE 2007 Heparin-binding EGF-like growth factor preserves mesenteric microcirculatory blood flow and protects against intestinal injury in rats subjected to hemorrhagic shock and resuscitation. Surgery $142: 234-242$

13. Barlow B, Santulli TV, Heird WC, Pitt J, Blanc WA, Schullinger JN 1974 An experimental study of acute neonatal enterocolitis-the importance of breast milk. J Pediatr Surg 9:587-595

14. Davis KM, Brigstock DR, Johnson PR, Crissman-Combs MA, McCarthy DW, Downing MT, Besner GE 1996 Production of glycosylated heparin-binding EGFlike growth factor in HeLa cells using vaccinia virus. Protein Expr Purif 8:57-67 
15. Caplan MS, Hedlund E, Adler L, Hsueh W 1994 Role of asphyxia and feeding in a neonatal rat model of necrotizing enterocolitis. Pediatr Pathol 14:1017-1028

16. Martin AE, Luquette MH, Besner GE 2005 Timing, route, and dose of administration of heparin-binding epidermal growth factor-like growth factor in protection against intestinal ischemia-reperfusion injury. J Pediatr Surg 40:1741-1747

17. Michalsky MP, Lara-Marquez M, Chun L, Besner GE 2002 Heparin-binding EGFlike growth factor is present in human amniotic fluid and breast milk. J Pediatr Surg 37:1-6

18. Feng J, Mehta VB, El-Assal ON, Wu D, Besner GE 2006 Tissue distribution and plasma clearance of heparin-binding EGF-like growth factor (HB-EGF) in adult and newborn rats. Peptides 27:1589-1596

19. Shin CE, Falcone RA Jr, Stuart L, Erwin CR, Warner BW 2000 Diminished growth factor levels in infants with necrotizing enterocolitis. J Pediatr Surg 35:173-176

20. Warner BB, Ryan AL, Seeger K, Leonard AC, Erwin CR, Warner BW 2007 Ontogeny of salivary epidermal growth factor and necrotizing enterocolitis. J Pediatr $150: 358-363$

21. Warner BW, Warner BB 2005 Role of epidermal growth factor in the pathogenesis of neonatal necrotizing enterocolitis. Semin Pediatr Surg 14:175-180

22. Miettinen PJ, Berger JE, Meneses J, Phung Y, Pedersen RA, Werb Z 1995 Epithelial immaturity and multiorgan failure in mice lacking epidermal growth factor receptor. Nature 376:337-341

23. Dvorak B, Halpern MD, Holubec H, Williams CS, McWilliam DL, Dominguez JA, Stepankova R, Payne CM, McCuskey RS 2002 Epidermal growth factor reduces the development of necrotizing enterocolitis in a neonatal rat model. Am J Physiol Gastrointest Liver Physiol 282:G156-G164

24. Vinter-Jensen L, Duch BU, Petersen JA, Ryslev A, Gregersen H 1996 Systemic treatment with epidermal growth factor in the rat. Biomechanical properties of the growing small intestine. Regul Pept 61:135-142

25. Sigalet DL, Martin GR, Butzner JD, Buret A, Meddings JB 2005 A pilot study of the use of epidermal growth factor in pediatric short bowel syndrome. J Pediatr Surg 40:763-768

26. Sullivan PB, Lewindon PJ, Cheng C, Lenehan PF, Kuo BS, Haskins JR, Goodlad RA, Wright NA, de la Iglesia FA 2007 Intestinal mucosa remodeling by recombinant human epidermal growth factor in neonates with severe necrotizing enterocolitis. J Pediatr Surg 42:462-469

27. Palomino A, Hernández-Bernal F, Haedo W, Franco S, Más JA, Fernández JA, Soto G, Alonso A, González T, López-Saura P 2000 A multicenter, randomized, doubleblind clinical trial examining the effect of oral human recombinant epidermal growth factor on the healing of duodenal ulcers. Scand J Gastroenterol 35:1016-1022

28. Dvorak B, Khailova L, Clark JA, Molla Hosseini D, Arganbright KM, Reynolds CA, Halpern MD 2008 Comparison of epidermal growth factor and heparin-binding epidermal growth factor-like growth factor for prevention of experimental necrotizing enterocolitis. J Pediatr Gastroenterol Nutr 47:11-18

29. 2005 Guidance for industry: Estimating the maximum safe starting dose in initial clinical trials for therapeutics in adult healthy volunteers. U.S. Department of Health and Human Services, Food and Drug Administration, Center for Drug Evaluation. Available at: http://www.fda.gov/CDER/GUIDANCE/5541fnl.htm 